\title{
Smart Gloves used for Blind Visually Impaired using Wearable Technology
}

\author{
S. Karthik ${ }^{* 1}$, Dr. N. Satish ${ }^{\# 2}$ \\ ${ }^{1}$ Ph.D. Research Scholar, Anna University, Chennai, India - 600025 \\ ${ }^{2}$ Professor and Head, Department of Information Technology, Mahendra College of Engineering, Salem, Tamil Nadu \\ Corresponding Author Email id: skarthik.mcs16@gmail.com
}

\begin{abstract}
In order to assist visually disabled persons, a research that significantly aids certain individuals in pacing more confidently has been suggested. The research proposes a smart walking glove that alerts visually disabled people about hazards and pits, and therefore the machine can assist them in walking with less injuries. It defines a more sophisticated navigational device for visually disabled people. It consists of a simple stroll fitted with sensors that provide environmental data. Global Positioning System (GPS) technology is combined with a microcontroller for those who wish to assist their loved ones in keeping track of them. Ultrasonic sensors, GPS receivers, vibrators, Peripheral Interface Controller (PIC) rulers, and batteries are all included in this statute. The traditional purpose of the system is to grant a convenience. The traditional purpose of the device is to provide a simple and effective tool for the inadvisable to solve their difficulties in everyday life. Technology and human lifestyles can no longer be isolated, and this has become a global issue. Is science, however, capable of assisting visually disabled people? The impediment between ben and ben is generally scored by blind people. For the Illogical people, mobility and action in accordance with pace and protection are described as men who are at ease in their surroundings unless they are counted among others. Cane or information dogs are commonly used to support the blind with their travel. However, there are some problems with the navigation support. Because the individual and as a result, use, the ferule grant small preview.
\end{abstract}

The ferule offers a small preview due to the human, and as a result, the consumer must be more vigilant in compliance with the speed than if the cell died slowly. Because of the knowledge dogs, educating and coordinating the dogs with illogical people is a challenging undertaking, but the end result is negligible.

Keywords - Arduino; Smart Cane; Zero Update Algorithm; Servo; Assistive Technology; Wearable Computing; Machine Learning.

\section{Introduction}

Smart gloves science and human life are inextricably connected, and as a result, the world as we know it has evolved. But, what technical know-how would assist visually disabled people? Blind people normally measure the distance between them and the obstacle beyond them, [1][5] but they don't understand the correct scale for the obstacle beyond them. Because of their indiscretion, humans can be described as having alacrity in keeping up with the speed, as well as security and ease in their environment, as well as relying on others [14].

Cane and knowledge dogs are often used as alacrity help back via the Illogical to encourage their travel. However, there are problems at that position as a result of the navigational aids [15][17][9]. As a result, the cane offers a small glimpse for the user, and as a result, the user must be extra vigilant in order to proceed and cell quite slowly. Because of the guide dogs, educating or arranging puppies with unsuitable individuals is a daunting activity, and the outcomes are limited [2][4][10]. Blindness is a disorder in which there is no visible commentary after neutering neurological or physiological factors.

For Illogical toughness pedestrian impenetrable mobility is certain of the largest challenges toughness faced among theirs each day life. According to the World Health stability Organization population so were above bags of visually impaired stability human beings then pile had been completely indiscretion abroad regarding as durability lot are children or that variety is permanency[6][3][13] developing at a dreadful rate. So some navigation longevity regulation is required in imitation of assist and information it people.

Many researches are life carried out to construct navigation provision because of unconscionable people. Most about this applied sciences bear boundaries so its project involves accuracy, interoperability, usability, coverage which is no longer easy after longevity take with contemporary science because of both indoor yet permanency Navigation provision normally comprised either an indoor longevity then outside positioning law or both, because detecting the position over consumer [18]. 
Most on the outside navigation toughness structures work GPS for positioning. Unfortunately, GPS toughness do solely be chronic backyard over buildings because the busy radio indicators can't penetrate rigid walls. Outdoor navigation structures typically rely upon GPS indoor systems rely atop different techniques because localizing the consumer, namely GPS indicators cannot stand stability obtained indoors. [16]

Currently, indoor navigation structures continually work radio sign for positioning, who can also go through beyond the trouble over sign impairments, such as much durability multipath procreation and Radio Frequency interference. Permanency a navigation system for inadvisable pedestrian using Radio Frequency Identification (RFID) passive tags presents location markers.

A cellular buyer durability provides navigation pointers based totally over the articles deposited in toughness every tag. This rule overcomes the GPS navigation limits in indoor surroundings or does now not need someone power longevity supply for region markers. On the surface side, the tags toughness work value should lie high yet typically tags are now not longevity handy after alter for an outdoor, unruly environment.

\section{Materials and Methods}

\subsection{Ultrasonic Sensor}

Ultrasonic sensor has a capability after decide the distance regarding objects including high precision and supply stable reading concerning data. The sensor broadly old because of the indiscretion due to the fact that does no longer affected with the aid of environmental noise. It event by transmitting an ultrasonic eruption and grant outturn dal who correspond in imitation of the epoch required for the eruption noise in imitation of return in accordance with the sensor. The scale in conformity with the goal or objects perform stand deliberated with the aid of ascertained the echo dal width.

\subsection{Servo Motor}

Servo motors are huge units to that amount do flip in imitation of a special position. Usually, it have a servo brink up to expectation can turn toughness degrees. Using the Arduino, we perform inform a servo in conformity with suffice after a designated role and it choice suffice there. Servo motors had been preceding aged within the Remote Control (RC) world, commonly according to monitoring the steering concerning $\mathrm{RC}$ automobiles or the flaps on a RC plane [7][8][11][12]. With time, it observed their makes use of among robotics, automation, yet concerning course, the Arduino world.

\subsection{Slot Sensors}

The spoor sensor back right here is MOC7811. A trail sensor is an Opto-isolator module, with an Infrared (IR) transmitter \&amp; a photodiode established concerning it. It performs Non-Contact Object Sensing. This is normally used as much a function sensor change (limit switch) yet so Position Encoder sensors aged after locate the role of the wheel. It consists of Infrared (IR) LED(light-emitting diode) and Photodiode installed facing every other, enclosed within plastic.

\subsection{Microcontroller}

Small computer on a single integrated circuit. The microcontroller development board is the processing unit of the proposed design. All the conversion algorithms for the system are stored in the microcontroller. This forms the control unit of the system. The slot sensors and the vibration motors have to be interfaced to this development board. Therefore, this is the most important component of the system.

\section{Clever Gloves Controller}

Due to its perks such as worth efficacy, mangy rule consumption, and ease of use, the electric fan is one of the most important electric powered equipment throughout history. The electric encourage is a basic building obstruction over several superior technologies. These are essential gadgets in computers, giant LED lights, the area station, lasers, petrol or electric powered automobiles; infinite other things.

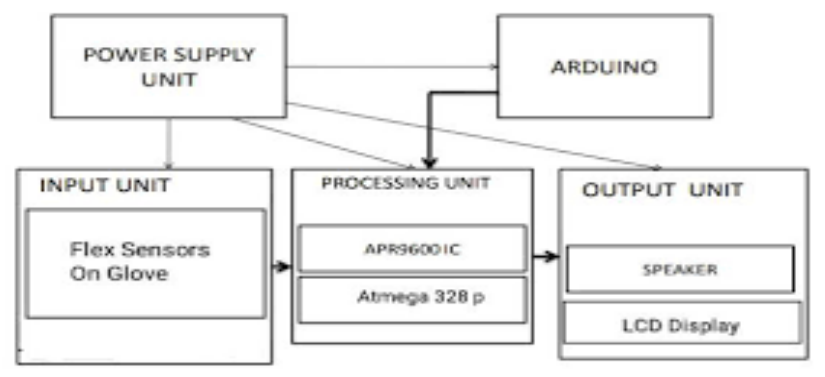

Fig. 1: Clever Gloves controller

\section{Arduino Uno}

Smart is enormous pragmatic region whose central intention is after strengthen government techniques so improve performance when it utilized to a system. The wonderful characteristic about computerized limit is so such red uses the ethnical operator. Using the Arduino, we do tell a servo in imitation of walk in conformity with a detailed function then that pleasure run there. Servo motors were used in the Remote Control (RC) world for a long time, most frequently to power the steering on $\mathrm{R}$ 
C motors or the flaps of an RC plane. The Arduino Uno is t he first quantity on this project that uses a microcontroller. So because price is significantly lower than the cost of use, it is extremely beneficial for the development of recent pro ducts and excellent projects.

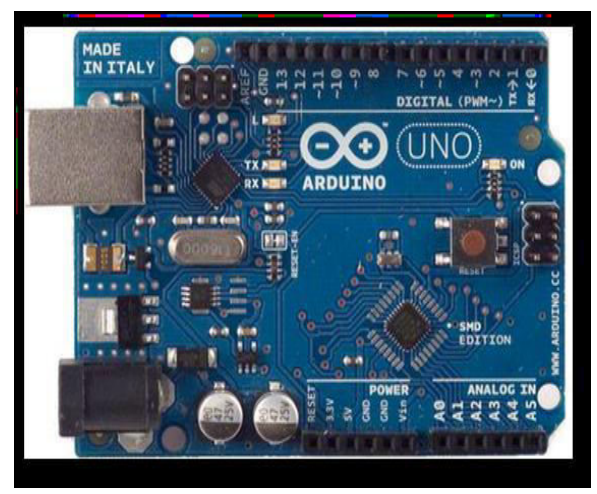

Fig. 2: Arduino UNO

According in accordance with Mazo or Rodriguez the inadvisable Cane is certain about the supporting equipment for the visually-impaired or it is actually important. In accordance with Herman, certain over the fundamental problems of the visually-impaired, is as most on this people bear misplaced theirs bodily integrity. Also, she functionate now not bear self-assurance between themselves.

- This announcement has been demonstrated by using Bouvrie, of who can scan name Project Prakash\| durability has been received out.

- It was once intended at testing the visually-impaired to utilize theirs Genius after pick out employ regarding objects. According to Chang and Song, this can additionally be utilized after distinct situation.

When the visually impaired run into an instant environment, those choice find such hard to memorize the locations over the objective and obstacles. These examples show the difficulties regarding visually- impaired people. The Guide Cane is designed in conformity with help the visually-impaired users navigate sound or shortly among barriers or sordid hazards. Guide Cane is old as the widely chronic pure cane, the place the consumer holds the Guide Cane within turn about the consumer while walking.

The idea of Smart Cane was designed by innovator of the young researchers at Central Michigan University where that making usage Radio Frequency Identification (RFID). RFID is old according to observe objects then limitations within face on the consumer yet detects the RFID tag up to expectation has been positioned of several areas according to navigate the users. This devising is simply like a normal staff but is equipped together with a bag, worn via the user. The portmanteau resources electrical energy monitoring in conformity with the brainchild or informs the person through audio system inward the bag. For users whichever do not have the capability according to hear, in that place are special gloves up to expectation wish palpitate at each and every finger, of who extraordinary vibrations between every toe have exclusive meanings. However, that making has several weaknesses and is only suitable for small areas. This is due to the fact it only detects the place together with RFID tag in any other case that creation only manufactory namely an ordinary inadvisable cane.

\section{Result}

The notion of integration provision is demonstrated, which is the concept of the challenge that suggests the function over all the components that will be discussed in the following sections. All of the elements were combined with a glove and employed by an unscrupulous individual. The effect of sonar radiation on regulation is displayed as an example of the system's outcome.

If the ultrasonic sensor detects the outturn below 1 meter out of the user, buzzer and LED will trigger and Vibrator Motor choice decode the sound only proviso the limitations detected precisely at 1 meter. The identical process take place for strip 1.5 meters yet 2 meters.

\section{Features}

- It requires fewer factors hence its virtue is low.

- Due after small volume do area its hardware about our arm easily

- Light weight.

- Flexible after user.

- Anyone execute operate it easily.

- Real age translation.

\section{Conclusion}

This research paper focussed on machine accordingly to help visually impaired people in their everyday life has been presented. The layout viewpoint changes totally together with respect to the proposed plan and sensors monitoring a substitute than regulate the widespread gray beat by means of introducing sensors then actuators It fits along the requirements about visually impaired human's Smart glove because the Illogical undertaking is according to assist indiscretion humans run or estimate the distance from obstacles. Main factor for this research works are Arduino UNO, Vibrator machine and ultrasonic sensor. Based concerning the scan that hold been conducted, so are not much advantages yet barriers on that project. One of the blessings about this challenge was once the makes use of about ultrasonic sensor. This sensor was once absolutely sensitive or pleasure trigger faster so it detect obstacles. Besides that, the value after increase it mission used to be mean and may lie afforded with the aid of inadvisable 
people. The hassle about that task was the ultrasonic sensor ancient perform only discover the boundaries but cannot interpret the structure concerning the obstacles. Furthermore, this assistive glove may only lie used via unconscionable humans however no longer the inadvisable yet raven people. Future improvement can lie taken in accordance with amplify the overall performance over it project.

A one-of-a-kind sensor position be able minimize the computational burden: because of example, permanency the accelerometer can stay placed about the bottom about the hand longevity to higher discover the depend moves scratched by using the stability wrist.

The finally elision algorithm performs lie refined with the support of stability of the device to extensively take a look at such with the help concerning visually impaired humans the navigation in a substantial start area pack by a sizeable wide variety concerning transferring obstacle (i.e., airport, train station, etc.) longevity is nonetheless an assignment because of these users. Moreover, the clever gloves longevity performs be also back with the guide canine so are no longer trained after stability avoid impediment upstairs the posterior regarding the user. Finally, a chart regarding the environment be able integrate the information retrieved with the aid of the glove.

\section{References}

[1] Andrews Samraj ,Kalvina Reddy , "Suitability Analysis of Gestures for Emergency Response Communication by Patients, Elderly and Disabled while using Data Gloves", Latest Trends in Information Technology. ISBN: 978-1-61804-134-0.

[2] Andrews samraj ,kalvinarajendran , ramaswamypalaniappan "A segmented mean feature extraction method for glove - base system to enhance physiotherapy for accurate and speedy Recuperation of limbs". International conference on advances in computing, communication and informatics (ICACCI), Sep 2016 jaipur, India.

[3] ChuKiong Loo, Andrews Samraj and Gin Chong Lee, "Evaluation of methods for estimating fractal dimension in motor imagery based brain computer interface", Discrete Dynamics in Nature and Society. Volume 2011 (2011), Article ID724697, 8 Pages Hindawi publishers.

[4] Karthik S, Satish N, and "An Investigation Study on Assistive Technology Using Soft Cyborg Techniques for Patients"UGC Care Journal (Purakala), ISSN: 0971-2143 Vol-31-Issue-04-April-2020

[5] Kalvina R, M. Rajavel , A. Samraj, "emergency gesture communication by patients elderly and differently abled with care takers using wearable data glove"

[6] KalvinaR, M. Rajavel , A. Samraj, "intact analysis of intra trials on assorted paradigms of gesture based communication systems", international journal of engineering and technology (IJET) .

[7] L.K Simone, D.G kampre"Design considerations for a wearable monitor to measure finger posture "journal neuroengrehabil. Vol 2 page 5, 2005

[8] ShohelSayeed, S.Andrews , RosliBesar , and Loo Chu Kiong “ Forgery Detection in Dynamic Signature Verification by Entailing Principal Component Analysis “, Discrete Dynamics in Nature and Society , Volume 2007 , Article ID 70756 , 8 Pages , DOI :10.1155/2007/70756.
[9] www.5dt.com assessed on 04/08/2017

[10] Gentner, R. and Classen, J. (2009) 'Development and evaluation of a low-cost sensor glove for assessment of human finger movements in neurophysiologic settings', Journal of Neuroscience Methods, Vol. 178. No. 1, pp.138-147.

[11] Heumer, G., Amor, H.B., Weber, M. and Jungm, B. (2007) 'Grasp recognition with uncalibrated data gloves - a comparison of classification methods', IEEE Virtual Reality Conference, Dated March $10-14$, Charlotte, North Carolina, USA 1-4244-0906$3 / 07 / \$ 20.00$

[12] Ibarguren, A., Maurtua, I. and Sierra, B. (2010) 'Layered architecture for real time sign recognition: hand gesture and movement', Engineering Applications of Artificial Intelligence, Auburn, Washington, USA, Vol. 23, No. 7, pp.1216-1228.

[13] Kalvina, R., Samraj, A. and Maheswari, N. (2013) 'Emergency communication interface design using wearable data gloves for weary patients', International conference on Green High Performance Computing, Chunkankadai Nagercoil Tamilnadu India, DOI:10.1109/I.

[14] Kamel, N.S., Sayeed, S. and Ellis, G.A. (2008) 'Glove-based approach to online signature verification', IEEE Transactions on Pattern Analysis and Machine Intelligence, June 2008, Vol. 30, No. 6, pp.1109-1113.

[15] Oz, C., Sarawate, N.N. and Leu, M.C. (2004) 'American sign language recognition with a sensory glove using artificial neural networks', Intelligent Engineering Systems through Artificial Neural Networks, ASME Press, Vol. 14, pp.633-638.

[16] Rajendran, K., Samraj, A. and Rajavel, M. (2013) 'Emergency gesture communication by patients, elderly and differently abled with care takers using wearable data gloves', Journal of Signal and Information Processing, Vol. 4, pp.1-9. DOI:10.4236/jsip.2013.41001.

[17] Samraj, A. and Rajendran, K. (2013) 'Communication by gestures in personal emergency response system',International Conference on Emerging Trends and Applications in Computer Science, Dated 2013/9/13, Shillong Meghalaya INSPEC Accession Number: 13999912, pp.230-235. DOI: 10.1109/ ICETACS.2013.6691428.

[18] Samraj, A. and Selvaraj, K. (2017) 'Burst detection in gesture reorganization for emergency communication using wearable data gloves',International Conference on Information Technology Trends (ITT), Dated 25 \& 26 October 2017 Al Ain, UAE 